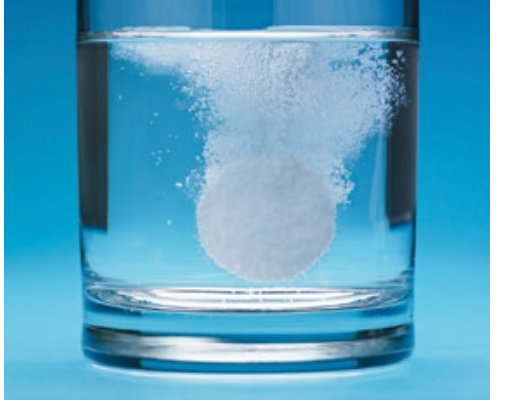

CPD

Jon D Emery, Peter Nguyen, Jesse Minshall, Kara-Lynne Cummings, Jennifer Walker

\section{Background \\ Prevention of cancer in primary care has focused on modifying behaviours associated with increased risk of cancer (primary prevention) or increasing participation in national cancer screening programs (secondary prevention). On the basis of meta- analyses of large prevention trials, a new paradigm in primary prevention - chemoprevention - is beginning to enter the realms of primary care for specific populations.}

\section{Objectives}

In this article, we discuss two examples of cancer chemoprevention relevant to general practice: low-dose aspirin for the prevention of colorectal cancer in people aged $50-70$ years, and selective oestrogen receptor modulators (SERMs) for women at increased risk of breast cancer. We present new expected frequency trees that show the absolute benefits and harms of taking these medications in specific populations.

\section{Discussion}

These expected frequency trees can serve as risk-communication aids to support shared decision making and the implementation of new chemoprevention guidelines in general practice.

\title{
Chemoprevention: A new concept for cancer prevention in primary care
}

UNTIL RECENTLY, cancer prevention in primary care has focused on influencing patient behaviours either by modifying lifestyle risks, such as smoking or alcohol consumption, or increasing participation in cancer screening programs (eg BreastScreen and the National Bowel Cancer Screening Program). Now a new paradigm, which involves using medications to reduce the risk of developing cancer, has entered general practice. General practitioners (GPs) regularly prescribe medications, such as statins or antihypertensive agents, to reduce a patient's risk of cardiovascular disease; however, prescribing drugs to reduce the risk of cancer is a new development in primary cancer prevention. In this paper, we explore the clinical implications of relatively new Australian guidelines on prescribing risk-reducing medication, or 'chemoprevention': specifically, aspirin to reduce colorectal cancer risk, ${ }^{1}$ and selective oestrogen receptor modulators (SERMs) to reduce breast cancer risk. ${ }^{2}$

\section{Aspirin and colorectal cancer prevention}

The strongest evidence for aspirin in reducing the risk of cancer arises from re-analysing individual-level patient data from seven cardiovascular prevention trials. ${ }^{3}$ This showed a 33\% reduction in all-cancer mortality after five years' follow-up, an effect that persisted to 20 years; the effect was greatest for gastrointestinal cancers, with a $35 \%$ reduction in mortality after 20 years. ${ }^{3}$ In 2017, Cancer Council Australia conducted a systematic review of the evidence to inform new guidelines specifically for colorectal cancer prevention. ${ }^{1}$ The evidence has not yet been considered to inform guidelines for preventing other cancers. Pooled data from four trials looking at aspirin for cardiovascular disease prevention showed a $25 \%$ reduced incidence of, and $33 \%$ reduced mortality from, colorectal cancer, with a median follow-up of 18.3 years. ${ }^{4}$ Interestingly, the effects of aspirin on colorectal cancer incidence were not observed until 10 years of follow-up. ${ }^{5}$ The benefits of aspirin are seen with low dosages that varied between trials from $100 \mathrm{mg}$ on alternate days to $300 \mathrm{mg}$ aspirin per day. There is some evidence from sub-group analyses of the pooled data that taking aspirin for only $2.5-5$ years may be as beneficial as consumption for more than five years. ${ }^{4}$

In summary, taking aspirin for as little as 2.5 years causes a delayed effect on colorectal cancer incidence, not seen until 10 years after initiation. The effects of aspirin on incidence and mortality appear to be specific to the proximal colon; ${ }^{4}$ tumours in this part of the gastrointestinal tract tend to present later and are more likely to be missed at colonoscopy. ${ }^{1}$ People at increased risk of colorectal cancer, either because of Lynch syndrome or a history of adenomas, are more likely to benefit than those at average risk of bowel cancer. ${ }^{6}$

The cancer-preventive effects of aspirin are in addition to the established 
benefits in reducing cardiovascular disease (myocardial infarcts, ischaemic strokes and transient ischaemic attacks). ${ }^{7}$ There are, of course, some limitations to the evidence. The trials were designed primarily to assess the effect of aspirin on cardiovascular outcomes rather than cancer. With the exception of the Women's Health Study (WHS), all the trials looking at aspirin for cardiovascular disease prevention were conducted in men. In the WHS, reduced colorectal cancer incidence was observed, but in modelling studies the cardiovascular benefit was greater than the reduction in colorectal cancer incidence. ${ }^{8}$ Aspirin has well recognised side-effects, including upper gastrointestinal symptoms and haemorrhagic stroke. However, fatal gastrointestinal bleeding rates did not differ between aspirin and placebo groups in pooled analyses. ${ }^{9}$ Overall, the modelled benefits significantly outweighed potential harms from aspirin. ${ }^{10}$ For example, it is estimated that for a person aged 50 years, taking aspirin for 10 years is 10 times more likely to prevent death than cause it, and five times more likely for someone aged 65 years. One death would be prevented for every 106 men aged 50 years and for every 46 men aged 65 years by taking aspirin for
10 years. ${ }^{10}$ Figure 1 presents expected frequency trees we have developed for an Australian population of 10,000 men and women aged 50-70 years, and shows likely outcomes over 10 years of taking aspirin for at least five years. ${ }^{10}$ Expected frequency trees are graphical summaries that aim to simplify multiple conditional probabilities and present the likelihood of specific outcomes. ${ }^{11}$

In November 2017, Cancer Council Australia published its Clinical practice guidelines for the prevention, early detection and management of colorectal cancer. ${ }^{1}$ These guidelines were endorsed by the National Health and Medical Research Council and recommend that GPs should actively consider prescribing low-dose aspirin (100-300 mg daily) to people aged $50-70$ years for a minimum of 2.5 years to reduce their risk of developing colorectal cancer. The Royal Australian College of General Practitioners' Guidelines for preventive activities in general practice 9th edition (Red Book) has recently been updated to reflect these new recommendations. The guidelines recommend that the choice to take aspirin is individualised, taking into account age, sex and cardiovascular risk. Of note, the US Preventive Services Task Force (USPSTF) currently only recommends aspirin for colorectal cancer prevention in people with at least a moderate $(10 \%)$ 10-year cardiovascular disease risk. ${ }^{12}$ The USPSTF recommendation is strongest for people aged 50-59 years, compared with those aged 60-69 years, because the risk of harms from aspirin increases with age. Recent findings from the ASPirin in Reducing Events in the Elderly (ASPREE) trial did not show any benefit of low-dose aspirin in people aged $>75$ years in terms of all-cause mortality or disability-free survival. ${ }^{13,14}$ However, this was after only a median of 4.7 years' follow-up and therefore too soon to expect any effect on cancer incidence or mortality. Precautions when prescribing aspirin include current dyspepsia or previous peptic ulcer, increased risk of gastrointestinal haemorrhage (eg current use of anticoagulants or other anti-platelets), bleeding diathesis, renal impairment or aspirin allergy.

\section{SERMs and breast cancer prevention}

Several randomised controlled trials have shown that SERMs (eg tamoxifen and raloxifene) significantly reduce the risk of breast cancer in women who have no personal history of the condition. ${ }^{15,16}$
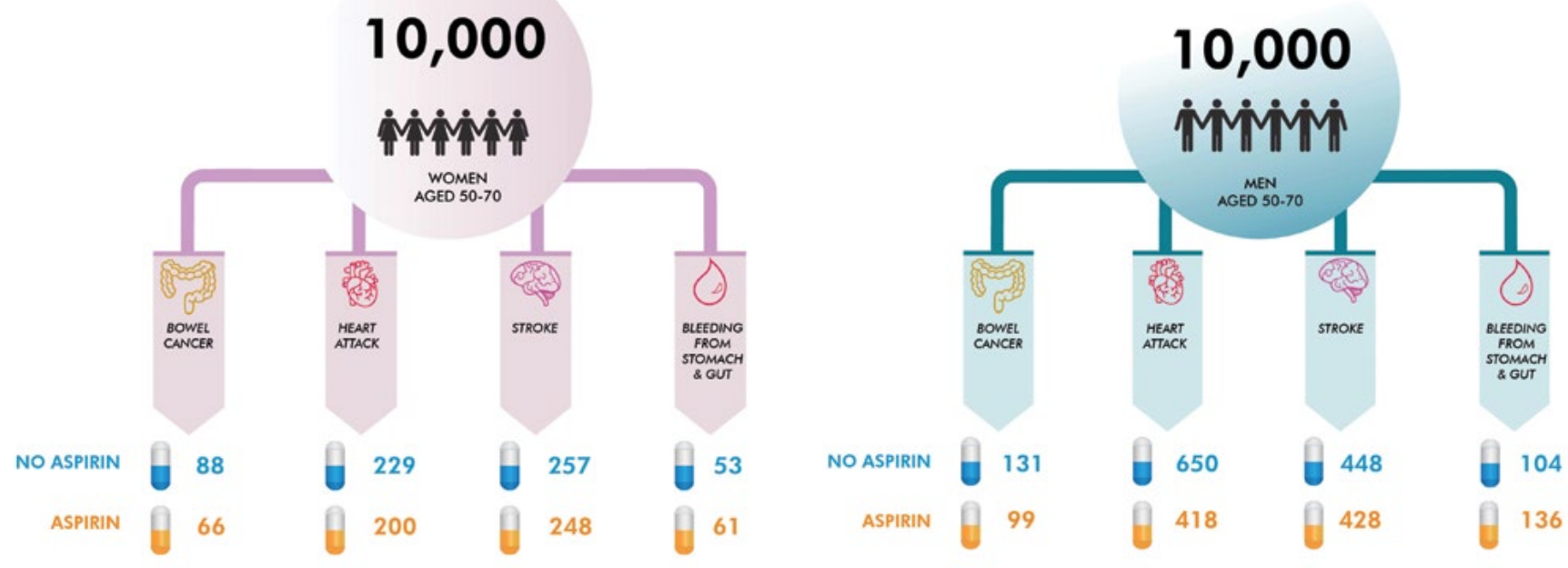

Figure 1. Expected frequency trees showing the effects of aspirin on the incidence of events over 10 years of taking aspirin for at least five years in Australian men and women aged $50-70$ years 
Meta-analyses of individual-level patient data from nine prevention trials show a $38 \%$ reduced incidence of breast cancer, but there were insufficient data to determine the effect on mortality. ${ }^{16}$ Populations studied in these trials included women at increased risk of breast cancer as well as those at average risk, and a wide range of age groups, both premenopausal and postmenopausal. The reduction in incidence extends beyond the period of taking the medication, providing ongoing protection for six to 10 years, although the effect is larger in the first five years of use. ${ }^{16}$ This effect is confined to oestrogen receptor-positive breast cancers and ductal carcinoma in situ (DCIS). Tamoxifen has a slightly greater effect on reducing the incidence of breast cancer than raloxifene, especially for DCIS, ${ }^{16}$ but the side effects of these drugs differ in important ways, which will influence drug choice.

Tamoxifen and raloxifene increase the risk of thromboembolic disease, although the effect is greater for tamoxifen. ${ }^{17}$ Tamoxifen also increases the risk of endometrial cancer. Common side effects of tamoxifen are vasomotor symptoms, and vaginal discharge and dryness; with raloxifene, common side effects are leg cramps and vasomotor symptoms. ${ }^{18}$ SERMs are not recommended in women with a past history of thromboembolic or cerebrovascular diseases, uninvestigated abnormal vaginal bleeding, or those who smoke or are on anticoagulants. ${ }^{2}$ Raloxifene is only used in postmenopausal women, whereas tamoxifen can be prescribed to either postmenopausal or premenopausal women who are not pregnant or breastfeeding. Through its therapeutic effect on osteoporosis, raloxifene has the additional benefit of reducing vertebral fractures. ${ }^{18}$ Figure 2 presents expected frequency trees we have developed for a hypothetical Australian population of women at moderate risk of breast cancer (relative risk 1.8) and outcomes of taking raloxifene or tamoxifen for five years, when compared with no treatment. ${ }^{16,19}$ We used Australian data on the incidence of venous thromboembolism ${ }^{20}$ and fractures, ${ }^{21}$ and data from the Study of Tamoxifen and Raloxifene (STAR) trial ${ }^{22}$ on effects on quality of life to estimate rates of these outcomes.

In 2011, Cancer Australia reviewed the evidence and recommended that tamoxifen (20 mg per day) or raloxifene
(60 mg per day) should be considered for women at moderate or high risk of breast cancer, based solely on family history criteria. ${ }^{2}$ Tamoxifen is now available on the Pharmaceutical Benefits Scheme (PBS) for this indication for a five-year preventive treatment period. The Red Book endorses these recommendations as an option for women at moderate or high risk of breast cancer.

\section{Summary}

The guidelines for aspirin and SERMs reflect a new approach to cancer prevention in primary care. The decision to prescribe these drugs involves careful discussion about the potential benefits and harms to an individual patient and consideration of underlying disease risks. The decision process may be more complex for SERMS than aspirin, given the greater side-effect profile and need to confirm a woman's risk of breast cancer. Women with a family history of breast cancer who are interested in exploring this option may be better referred to a familial cancer clinic or specialist breast cancer service. However, the discussion about taking aspirin could sit well within
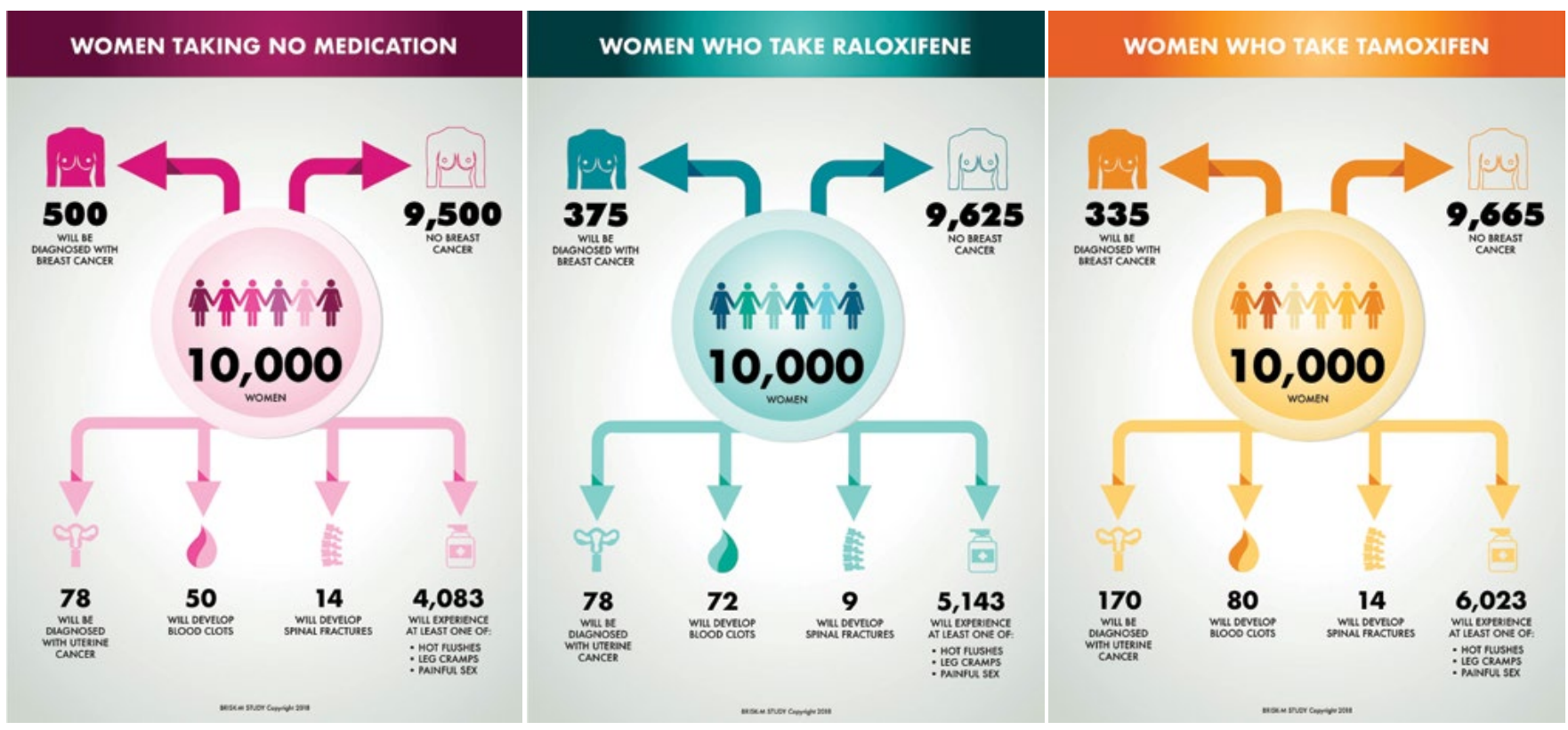

Figure 2. Expected frequency trees for Australian females at moderate risk of breast cancer, showing the effects of taking tamoxifen and raloxifene for five years 
primary care. The expected frequency trees presented in this paper could support shared decision making and allow patients to decide whether to use this approach to reduce their risk of cancer. Of course, this approach should not be considered as an alternative, but as an addition to lifestyle modification to prevent cancer.

\section{Authors}

Jon D Emery MA, MBBCh, FRACGP, MRCGP, DPhil, Herman Professor of Primary Care Cancer Research, Centre for Cancer Research and Department of General Practice, Faculty of Medicine, Dentistry and Health Sciences, University of Melbourne, Vic. jon.emery@unimelb.edu.au

Peter Nguyen BBiomed(Hons) student, Centre for Cancer Research and Department of General Practice, Faculty of Medicine, Dentistry and Health Sciences, University of Melbourne, Vic

Jesse Minshall MD, BSc, medical intern, Western Health, Footscray, Vic

Kara-Lynne Cummings BA, PC4 Project Officer, Centre for Cancer Research and Department of General Practice, Faculty of Medicine, Dentistry and Health Sciences, University of Melbourne, Vic Jennifer Walker BAppSci, MPH, PhD, Senior Research Fellow, Centre for Cancer Research and Department of General Practice, Faculty of Medicine, Dentistry and Health Sciences, University of Melbourne, Vic

Competing interests: None.

Funding: JDE is funded by an NHMRC Practitioner Fellowship.

Provenance and peer review: Commissioned, externally peer reviewed.

\section{References}

1. Cancer Council Australia. Clinical practice guidelines for the prevention, early detection and management of colorectal cancer. Sydney: Cancer Council Australia, 2017.

2. Cancer Australia. Risk reducing medication for women at increased risk of breast cancer due to family history. Sydney: Cancer Australia, 2011.

3. Rothwell PM, Fowkes FG, Belch JF, Ogawa H, Warlow CP, Meade TW. Effect of daily aspirin on long-term risk of death due to cancer: Analysis of individual patient data from randomised trials. Lancet 2011;377(9759):31-41. doi: 10.1016/S01406736(10)62110-1.

4. Rothwell PM, Wilson M, Elwin CE, et al. Long-term effect of aspirin on colorectal cancer incidence and mortality: 20-year follow-up of five randomised trials. Lancet 2010;376(9754):1741-50. doi: 10.1016/S0140-6736(10)61543-7.

5. Peto R, Gray R, Collins R, et al. Randomised trial of prophylactic daily aspirin in British male doctors. Br Med J (Clin Res Ed) 1988;296(6618):313-16.

6. Cole BF, Logan RF, Halabi S, et al. Aspirin for the chemoprevention of colorectal adenomas: Meta-analysis of the randomized trials. J Nat Cancer Inst 2009;101(4):256-66. doi: 10.1093/ jnci/djn485.

7. Antithrombotic Trialists' (ATT) Collaboration, Baigent C, Blackwell L, et al. Aspirin in the primary and secondary prevention of vascular disease: Collaborative meta-analysis of individua participant data from randomised trials. Lancet 2009;373(9678):1849-60. doi: 10.1016/S01406736(09)60503-1.
8. van Kruijsdijk RC, Visseren FL, Ridker PM, et al. Individualised prediction of alternate-day aspirin treatment effects on the combined risk of cancer, cardiovascular disease and gastrointestinal bleeding in healthy women. Heart 2015;101(5):369-76. doi: 10.1136/ heartjnl-2014-306342.

9. Chubak J, Kaminieni A, Buist DSM, Anderson ML, Whitlock EP. Aspirin use for the prevention of colorectal cancer: An updated systematic evidence review for the US Preventive Services Task Force [Internet]. Rockville, MD: Agency for Healthcare Research and Quality (US), 2015.

10. Cuzick J, Thorat MA, Bosetti C, et al. Estimates of benefits and harms of prophylactic use of aspirin in the general population. Ann Oncol 2015;26(1):47-57. doi: 10.1093/annonc/mdu225.

11. Spiegelhalter D, Pearson M, Short I. Visualizing uncertainty about the future. Science 2011;333(6048):1393-400. doi: 10.1126/ science.1191181.

12. US Preventive Services Task Force. Final recommendation statement: Aspirin use to prevent cardiovascular disease and colorectal cancer: Preventive medication. Rockville, MD: USPSTF, 2017.

13. McNeil JJ, Nelson MR, Woods RL, et al. Effect of aspirin on all-cause mortality in the healthy elderly. N Engl J Med 2018;379(16):1519-28. doi: 10.1056/ NEJMoa1803955.

14. McNeil JJ, Woods RL, Nelson MR, et al. Effect of aspirin on disability-free survival in the healthy elderly. N Engl J Med 2018;379(16):1499-508. doi: 10.1056/NEJMoa1800722.

15. Cuzick J, DeCensi A, Arun B, et al. Preventive therapy for breast cancer: A consensus statement. Lancet Oncol 2011;12(5):496-503. doi: 10.1016/ S1470-2045(11)70030-4.

16. Cuzick J, Sestak I, Bonanni B, et al. Selective oestrogen receptor modulators in prevention of breast cancer: An updated meta-analysis of individual participant data. Lancet 2013;381(9880):1827-34. doi: 10.1016/S01406736(13)60140-3.

17. Visvanathan K, Hurley P, Bantug E, et al. Use of pharmacologic interventions for breast cancer risk reduction: American Society of Clinical Oncology clinical practice guideline. J Clin Oncol 2013;31(23):2942-62. doi: 10.1200/ JCO.2013.49.3122.

18. US Preventive Services Task Force. Final recommendation statement: Breast cancer: Medications for risk reduction. Rockville, MD: USPSTF, 2016

19. Freedman AN, Yu B, Gail MH, et al. Benefit/risk assessment for breast cancer chemoprevention with raloxifene or tamoxifen for women age 50 years or older. J Clin Oncol 2011;29(17):2327-33. doi: 10.1200/JC0.2010.33.0258.

20. Ho WK, Hankey GJ, Eikelboom JW. The incidence of venous thromboembolism: A prospective, community-based study in Perth, Western Australia. Med J Aust 2008;189(3):144-47.

21. Sanders KM, Seeman E, Ugoni AM, et al. Ageand gender-specific rate of fractures in Australia: A population-based study. Osteoporos Int 1999;10(3):240-47. doi: 10.1007/s001980050222.

22. Vogel VG, Costantino JP, Wickerham DL, et al. Update of the National Surgical Adjuvant Breast and Bowel Project Study of tamoxifen and raloxifene (STAR) P-2 trial: Preventing breast cancer. Cancer Prev Res (Phila) 2010;3(6):696-706. doi: 10.1158/1940-6207.CAPR-10-0076. 